\title{
Motor Area Activity During Mental Rotation Studied by Time-Resolved Single-Trial fMRI
}

\author{
Wolfgang Richter, Ray Somorjai, Randy Summers, \\ and Mark Jarmasz \\ National Research Council, Manitoba, Canada
}

\section{Ravi S. Menon and Joseph S. Gati}

Robarts Research Institute, Ontario, Canada

\section{Apostolos P. Georgopoulos}

Veterans Affairs Medical Center, Minnesota

\author{
Carola Tegeler, Kamil Ugurbil, and Seong-Gi Kim \\ University of Minnesota Medical School
}

\begin{abstract}
The functional equivalence of overt movements and dynamic imagery is of fundamental importance in neuroscience. Here, we investigated the participation of the neocortical motor areas in a classic task of dynamic imagery, Shepard and Metzler's mental rotation task, by time-resolved single-trial functional Magnetic Resonance Imaging (fMRI). The subjects performed the mental-rotation task 16 times, each time with different object pairs. Functional images were acquired for each pair separately, and the onset times and
\end{abstract}

\section{INTRODUCTION}

An ever recurring and yet unproven hypothesis in the neuroscientific literature is the functional equivalence of dynamic imagery and overt movements (Weimer, 1977; James, 1950). While there is a wealth of experimental data on the brain structures subserving the latter, analogous experiments concerning dynamic imagery are generally much less straightforward to design and to interpret. A frequently studied example of a dynamic imagery task is the mental rotation of 3-D objects in space, originally introduced by Shepard and Metzler (1971) (Figure 1). The task is to decide whether two objects shown are identical to one another or mirror images; in general, the two objects are rotated relative to each other. It was conjectured that the subject carries out this task by rotating the objects along a smooth angular trajectory into congruence with one another. Evidence for this comes from psychophysical studies. Most important is the fact that the response time (RT) increases linearly with the rotation angle, thus suggest- widths of the activation peaks in each area of interest were compared to the response times. We found a bilateral involvement of the superior parietal lobule, lateral premotor area, and supplementary motor area in all subjects; we found, furthermore, that those areas likely participate in the very act of mental rotation. We also found an activation in the left primary motor cortex, which seemed to be associated with the right-hand button press at the end of the task period.

ing that the object is rotated mentally with constant angular velocity (Shepard \& Metzler, 1971).

It is well-known that the superior-parietal lobule is involved in mental rotation (Tagaris et al., 1996; Tagaris et al., 1997; Tagaris et al., 1998; Alivisatos \& Petrides, 1996; Cohen et al., 1996). However, studies of the activity in motor areas in the visual-mental rotation task under investigation here have been somewhat less conclusive. Previous fMRI studies of the mental rotation of 3D objects in our laboratory showed that the activation in the right precentral gyrus is related to the rate of mental rotation, whereas activation in the superior parietal lobule is related to the performance (Tagaris et al., 1996; Tagaris et al., 1997). Similarly, the precentral gyrus was shown to be activated bilaterally during the performance of 2-D visual mental rotation (Tagaris et al., 1998). A recent study employing positron emission tomography (PET) compared the mental rotation of Shepard and Metzler's figures with the mental rotation of images of hands (Kosslyn, DiGirolamo, Thompson, \& 


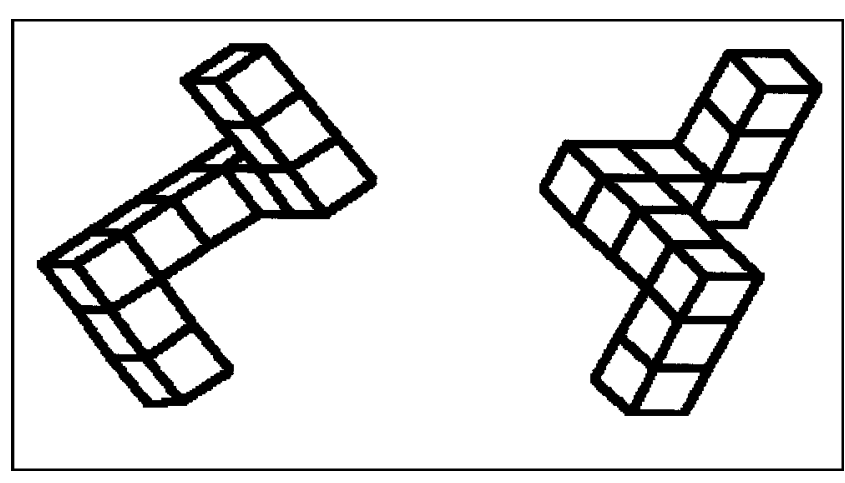

Figure 1. Shepard and Metzler's mental rotation task. The subject has to decide whether the two objects are identical or mirror images; in this case, the objects are identical.

Alpert, 1998). Even though both tasks activated areas throughout the parietal lobe, the rotation of the abstract objects did not induce an activation in the frontal motor areas. The authors concluded that Shepard and Metzler's task, unlike the rotation of hand images, does not involve low-level motor processes. This result may be compared to an earlier fMRI study (Cohen et al., 1996), where the authors found consistent activation only in the supplementary motor area (SMA), but not in other frontal motor areas. The activation in SMA was tentatively attributed to the attentional requirement of this complex task. A study of Parkinson's disease (PD) found that 3-D, but not 2-D, mental rotation is impaired in patients; however, the authors did not suggest that the motor deficits accompanying PD are causally related to the mental-rotation impairment (Lee, Harris, \& Calvert, 1997). An EEG study of the 3-D mental rotation found activity over left premotor regions and other areas of the frontal cortex (Williams, Rippon, Stone, \& Annett, 1995). The authors concede that this may be due not only to an isolated-motor process, but also to some interactions with attentional or decision-making processes.

In the present experiment, we investigated the functional activity in the frontal motor areas during the performance of the mental-rotation task by time-resolved fMRI (Richter, in press; Kim, Richter, \& Ugurbil, 1997; Richter, Andersen, Georgopoulos, \& Kim, 1997a; Richter, Ugurbil, Georgopoulos, \& Kim, 1997b). At high magnetic fields, there is often enough sensitivity to monitor the evolution of the fMRI signal in a single execution of a cognitive task without averaging over many trials. Hence, it is possible to perform separately many such single trial executions of a task. Subsequently, temporal characteristics (such as onset time and width) of the fMRI signal can be correlated with behavioral data, such as the response time. A schematic of this method is shown in Figure 2. For example, in our previous investigation of motor tasks (Kim et al., 1997; Richter et al., 1997b), the fMRI responses in the motor areas were compared with the well-controlled
Figure 2. Schematic description of time-resolved fMRI. The straight lines symbolize linear fits of a time-course parameter to a behavioral parameter. If the two parameters are correlated, we may conclude that the observed activity is caused by a neuronal event that scales with the behavioral parameter (i.e., the response time). If they are not correlated, the activity is caused by an event that is constant from trial to trial. Depending on the nature of the task, the behavioral parameter, and the fMRI time-course parameters, conclusions may be drawn about the temporal sequence of neuronal events on a time scale shorter than the range of possible hemodynamic responses.

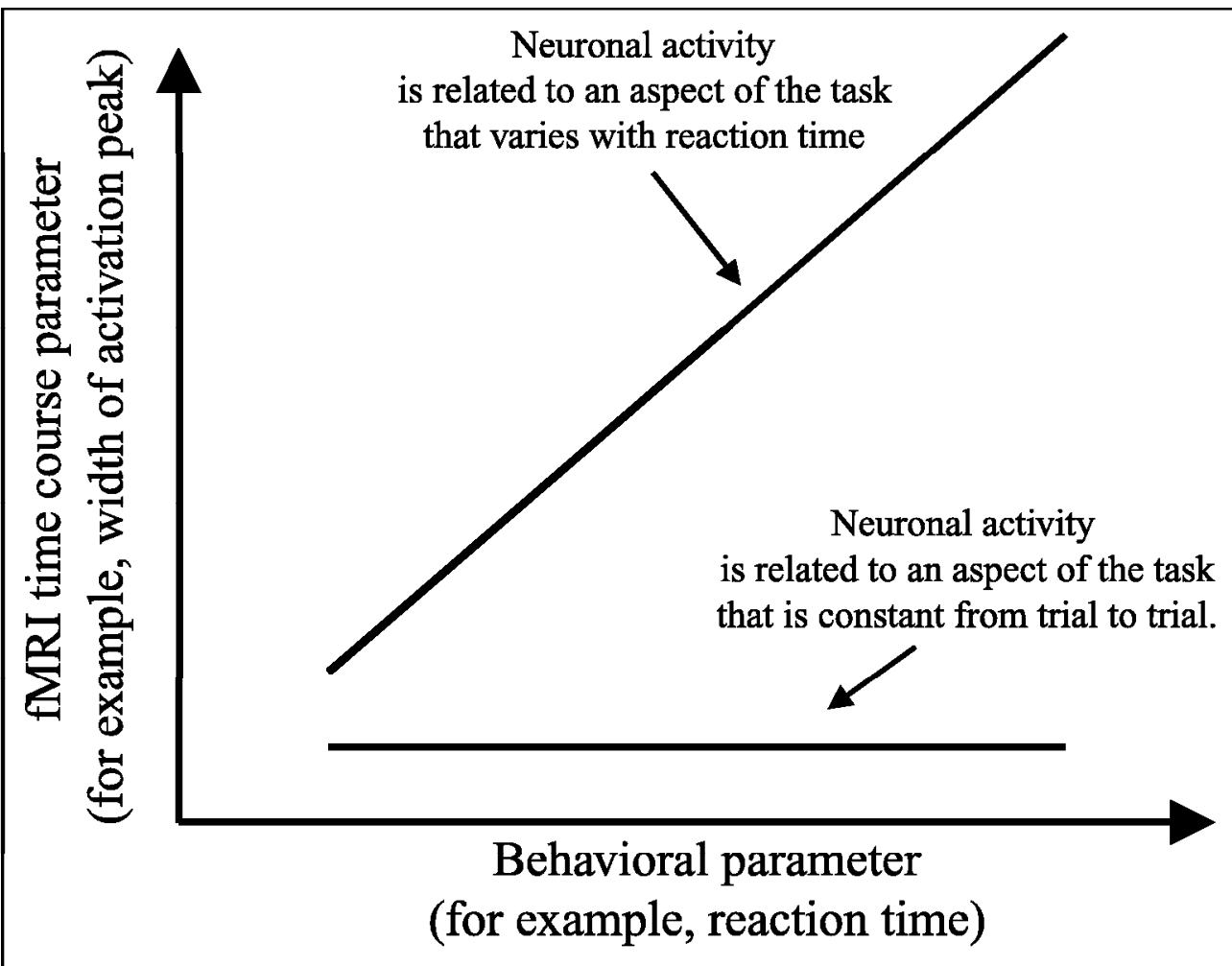


motor preparation times. In that study, the premotor area (PM) and the SMA were found to be activated during both the motor preparation and execution periods. Our group has also used this method (Richter et al., 1997b) to investigate the participation of the superior parietal lobe (SPL) in the execution of the mental-rotation task. We found that the onset of the fMRI signal was independent of the response time (the time the subject takes to make a decision), whereas its duration (minus a constant offset) was equal to the response time. We concluded that the SPL is intimately involved in the very act of mental rotation. That experiment showed that the specific function of a given brain area in a cognitive task can be determined by this method, beyond merely stating whether the area is activated or not. Here, we investigate the participation of neocortical motor areas in the mental rotation task.

\section{RESULTS}

Shepard and Metzler (1971) found a linear increase of the response time with the rotation angle in their mental rotation task. Therefore, we assessed the linear- ity of this function for our data in order to verify that the subjects were indeed carrying out this task by the same process. However, the statistical power is much lower (we only used 10 identical object pairs in each subject, as compared to 400 in Shepard and Metzler's original experiment). Furthermore, each object occurred only twice among these 10 pairs (five different objects and their mirror images). We found an overall confidence level of $p=.044$ for a linear correlation of the rotation angle and response time. The rotation-speed average over all objects was found to be approximately $20^{\circ} / \mathrm{sec}$. This is considerably lower than Shepard and Metzler's result of $50^{\circ} / \mathrm{sec}$. We suspect that the reason for this discrepancy lies in the instruction we gave the subject to try to solve the problem correctly, regardless of speed. The mechanism of rotation might, therefore, be different, or the subjects might carry out the rotation more than once.

A parametric-activation map for the second slice from the top in one subject is shown in Figure 3. Note that not all areas that show activation, such as the frontal eye fields, were considered further for the purpose of this experiment. In all six subjects, bilateral activation was found in the SPL, in the lateral premotor areas (lateral

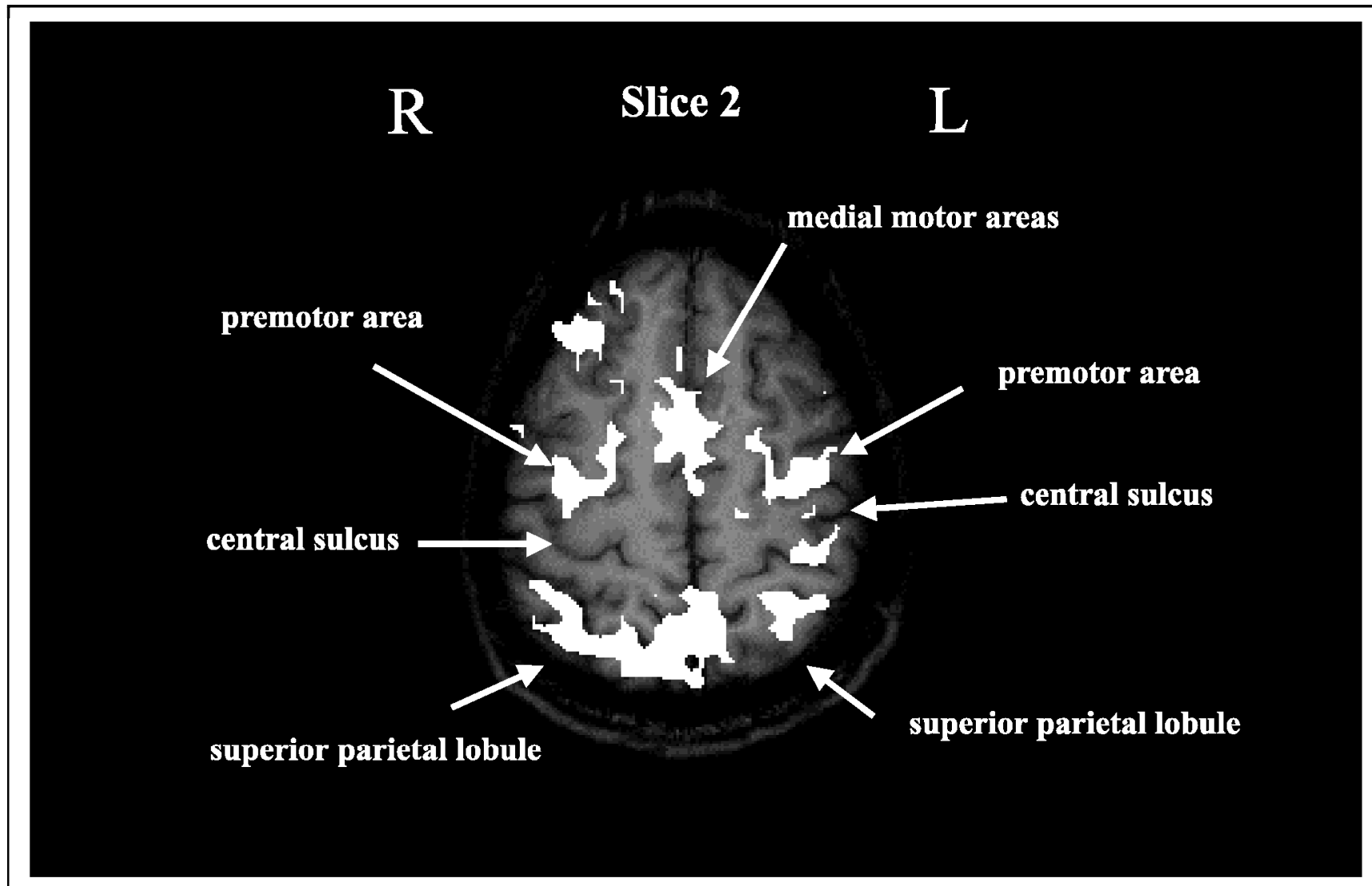

Figure 3. Parametric simultaneous activation map for one subject (one slice, all trials). This map is interpolated from the calculated map's $64 \times 64$ matrix size to the anatomical background $(256 \times 256)$. The activation can be seen in the lateral and medial motor areas, in the superior parietal lobule, and in the other cortical areas. Note that the activation is not always confined to anatomically well-defined areas; in this study, only the activated pixels in SPL and motor areas, as defined in the text, were considered for further analysis. 
Figure 4. Concatenated averaged time courses for 16 trials, showing the activation from 69 voxels in the SMA (one subject). The black triangles indicate the beginning of each task period.

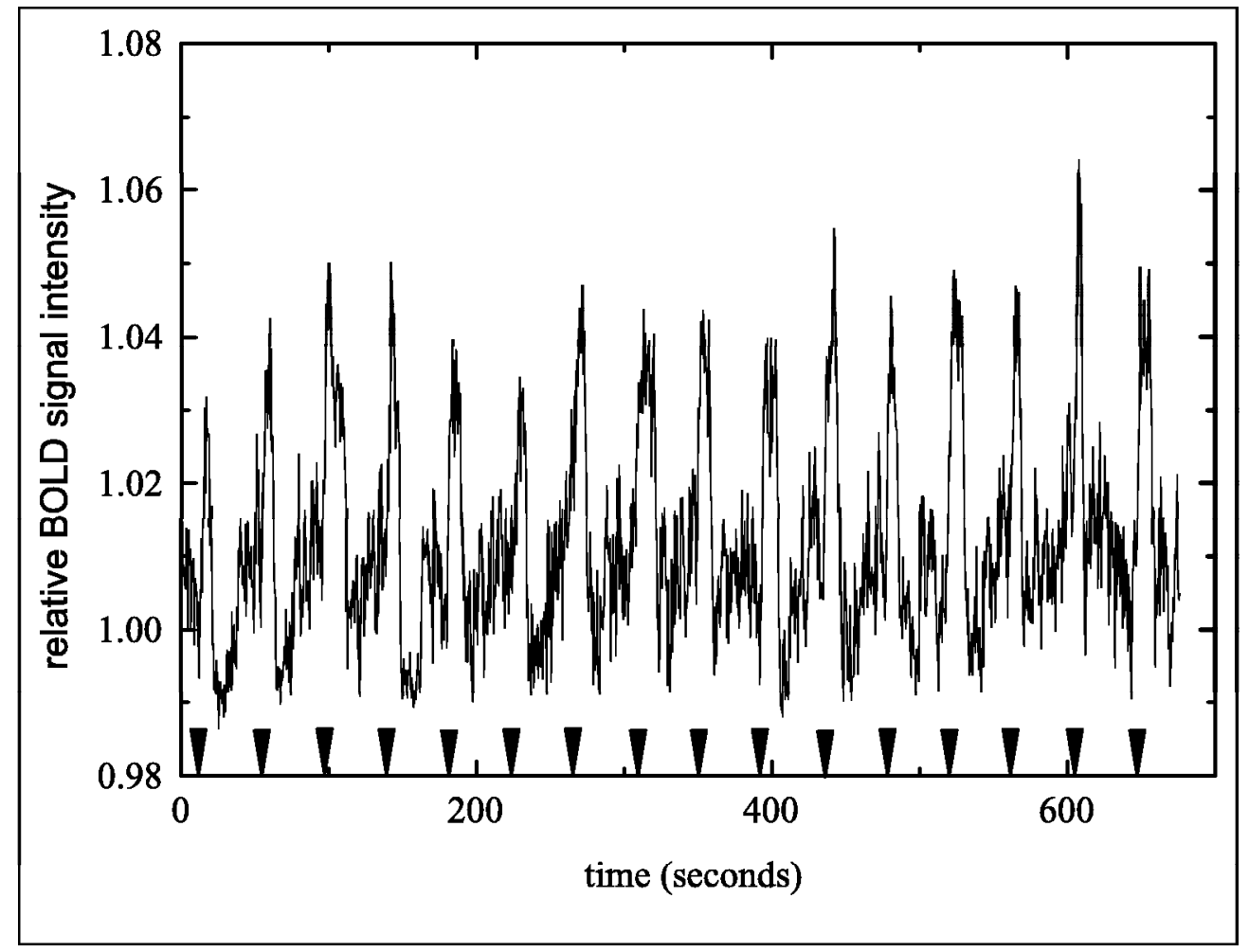

BA 6), and the SMA (medial BA 6). Activation was also found in the left M1. Furthermore, we observed an activation in the right M1 (BA 4) in five out of six subjects. In comparison with our results, a previous fMRI study (Cohen et al., 1996) found activation in the SMA in four out of eight subjects, and in the right BA 4 in
Figure 5. Averaged time courses in the right M1 (upper curve) and SMA (lower curve) in one subject. The average is time-locked to the response time.

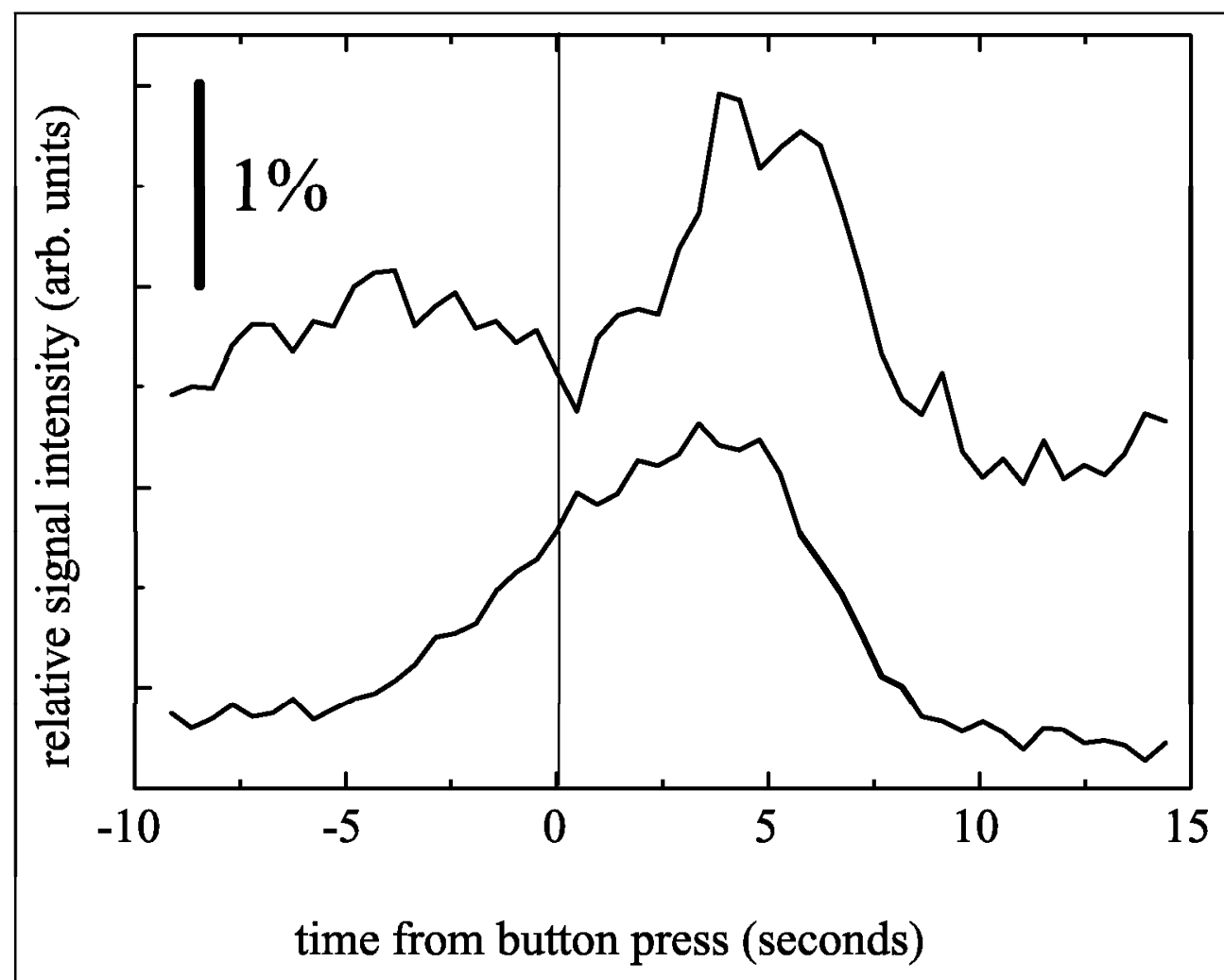


Figure 6. Graph of the width (solid circles) and onset (crosses) vs. the response time for one subject in the SMA (16 trials). The width and response time are significantly correlated $(y=1.04 x+.9 \mathrm{sec}$; $p<.0001)$; the onset and response time are not significantly correlated.

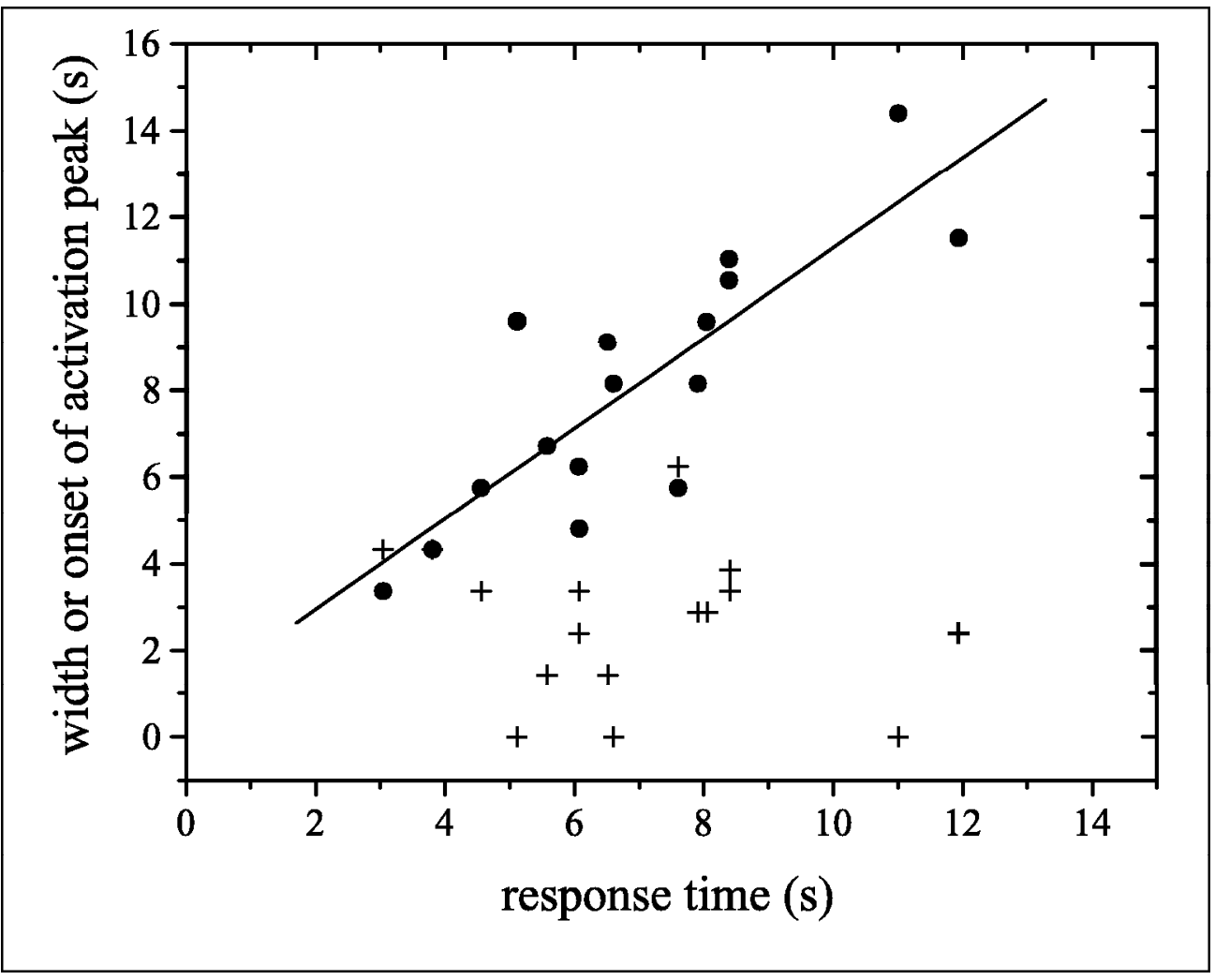

two out of eight subjects. Shown in Figure 4 is the average time course from 69 activated voxels in the SMA in one subject, from 16 concatenated trials; the beginning of the task period in each trial is marked by a triangle at the bottom of the graph. Note that there are 16 activation peaks, one for each of the 16 pairings.

The main goal of our study was to determine the specific role of the motor areas in this task. We also included the SPL in the analysis, which we had studied previously, though using a different slice orientation and, hence, covering a somewhat different area (Richter et al., 1997b). Consequently, seven regions of interest (ROIs) were analyzed separately: Left and right SPL, SMA, left and right premotor areas, and left and right M1. Average time courses of activated pixels, as determined by a nonparametric analysis, were calculated for each ROI in each subject.

In Figure 5, we display the response-time-locked average time courses from one subject (16 trials). The upper curve displays the time course from the left M1, the lower one from the SMA. These time courses are averaged over all 16 trials such that the response times coincide at time index "0." Qualitatively, we can see that, in M1, the BOLD signal rises after the button press and is narrow by comparison. In the SMA, on the other hand, the leading edge of the peak increases gradually before the button press; the trailing edge coincides approximately with that of the signal from M1. This means qualitatively that the signal onset in M1 is a monotonic or even linear function of the response time, leading to constructive averaging when the time courses are shifted by the response time. In the SMA, the signal onset then seems to be independent of the response time, while the trailing edge of the peak is, again, a monotonic function of the response time.

In order to quantitate these observations, we defined two time-course parameters for each single trial: the onset of activation, and the width of the activation waveform. In Figure 6, we show the correlation between the onset/width of the SMA and the response time for all 16 experiments in one subject. Note that the width and response time are positively correlated; the onset and

Table 1. Number of Subjects for Whom the Onset/Width are Significantly $(p<.05)$ Correlated with the Response Time

\begin{tabular}{lcc}
\hline & Onset & Width \\
\hline Left M1 & 1 & 1 \\
Left parietal & 1 & 2 \\
Left premotor & 0 & 5 \\
SMA & 0 & 4 \\
Right M1 & 0 & 0 \\
Right parietal & 0 & 3 \\
Right premotor & 0 & 4 \\
\hline
\end{tabular}


Table 2. Parameters of the Linear Fit of the Onset and Width vs. the RT Across All Subjects

\begin{tabular}{|c|c|c|c|c|c|c|}
\hline & \multicolumn{3}{|c|}{ Onset } & \multicolumn{3}{|c|}{ Width } \\
\hline & Slope & Intercept (sec) & p value & Slope & Intercept (sec) & p value \\
\hline Left M1 & 0.61 & 1.7 & 0.0002 & 0.10 & 5.40 & $>0.1$ \\
\hline Left SPL & -0.03 & 3.1 & $>0.1$ & 0.65 & 5.0 & $2 \times 10^{-6}$ \\
\hline Left premotor & -0.05 & 2.5 & $>0.1$ & 0.96 & 2.2 & $<10^{-12}$ \\
\hline SMA & -0.04 & 2.6 & $>0.1$ & 0.88 & 3.1 & $1 \times 10^{-6}$ \\
\hline Right M1 & -0.28 & 10.5 & $>0.1$ & -0.07 & 5.8 & $>0.1$ \\
\hline Right SPL & -0.05 & 3.2 & $>0.1$ & 0.79 & 4.4 & $1 \times 10^{-5}$ \\
\hline Right premotor & -0.09 & 3.5 & $>0.1$ & 0.92 & 3.0 & $3 \times 10^{-6}$ \\
\hline
\end{tabular}

Significant values $(p<.05)$ are shown in bold.

response time are uncorrelated. We performed this analysis for all subjects and ROIs; we also calculated the $p$ value for the null hypothesis that the onset or width is independent of the response time. Shown in Table 1 is the number of subjects for whom the onset or width and response time are found to be correlated (i.e., for whom we could reject the null hypothesis of no correlation).

In Table 2 , the slopes, $y$-intercepts and $p$ values for the linear fit of the onset/width vs. RT are shown for all subjects combined. We found that in the left M1, the onset and response time were significantly correlated with one another $(p<.05)$, and that in all other areas the width and response time were significantly correlated, with the exception of the right M1, which showed no significant correlation at all.

\section{DISCUSSION}

\section{Technical Considerations}

Since a single image can be obtained within $50 \mathrm{msec}$, fMRI can detect the hemodynamic response induced by neural activity in real time. To obtain meaningful hightemporal resolution fMRI data, several approaches have been used in the past: An "averaged single trial" method (Buckner et al., 1996), a mixed-trial technique (Clark, Maisog, \& Haxby, 1998), and the general "TrialBased" (TB) design (Zarahn, Aguirre, \& D'Esposito, 1997). Our method is a true single-trial technique (Richter, in press; Kim et al., 1997; Richter et al., 1997a; Richter et al., 1997b), and a subset of all possible TB designs. In the averaged single trial method, the fMRI signal acquisition was gated by the onset of the presentation of some task, independent of behavior. That approach is permissible in the limit where behavior and brain function do not vary significantly over repeated trials; this may limit the applicability to some experiments. In addition, insofar as intrinsic hemodynamic responses may be different in different regions, information about temporal differences of neural activity is necessarily limited. For example, it has been observed that the onset of the activation in the left prefrontal cortex was delayed by about $1 \mathrm{sec}$ relative to that in the extrastriate areas during the performance of word generation, and that it decayed considerably more slowly (Buckner et al., 1996). Since the difference in the onsets of neuronal activity between the two areas is known to be only a few hundred milliseconds, the difference in the fMRI responses may be related to the difference in the intrinsic hemodynamic responses (Buckner et al., 1996). Thus, comparing the time courses across functionally specialized regions is sometimes problematic. Hence, sequential neuronal activity cannot be easily determined simply from the difference in onset times in single averaged time courses. In spite of this difficulty, however, this approach is extremely valuable if information is sought on a temporal scale more coarse than the variations in hemodynamic response times. The mixed-trial technique (Clark et al., 1998) uses a sequence of random stimuli, presented in rapid succession; the data are then analyzed by correlating the responses with the specific sequence pattern of each individual stimulus type. This method makes it possible to assign the activation in different areas of the brain to different tasks or aspects of a task, but, again, the temporal resolution is limited to the possible differences in the hemodynamic response times between regions. The general class of $T B$ designs exploits the ability to detect signal changes subsequent to isolated single trials; it can, in the words of Zarahn et al., "utilize intertrial variance in uncontrolled behavioral measures to examine their functional correlates" (Zarahn et al., 1997). While Zarahn et al. suggested and used discretely varying parameters (like the handedness of a motor output), our experiment uses a continuously varying parameter (the reaction time) as the behavioral measure. 
Time-resolved fMRI is a unique complement to the other techniques mentioned, because hemodynamicresponse time differences between regions are canceled through the use of multiple individual trials.

Since no averaging over several executions of a task is performed in time-resolved single-trial fMRI, SNR can be poor. Thus, this method may often not be feasible, particularly at low magnetic fields. However, even if the sensitivity is not sufficient for the detection of a single-task execution, it would still be possible to perform many such single-trial executions of a task separately. Selective signal averaging can then be employed, using a behavioral parameter (for example, the response time) as a criterion. An example of this approach was shown in Figure 5. Then, temporal characteristics (such as onset time and width) of the fMRI responses could still be correlated with behavioral data such as the response time (Menon, Luknowsky, \& Gati, 1998). In this way, differences in the hemodynamic response time could be subtracted out and distinguished from the temporal behavior of neuronal activity itself (Menon \& Kim, 1999). Hence, this approach permits the determination of the specific function of given brain areas in the context of a single complex task, unlike the methods using indiscriminately averaged single time courses.

We concede that one of the main assumptions of time-resolved fMRI is that the fMRI response is a linear function of the neuronal activity. This is indeed suggested by various studies; for example, a linear response was measured in the visual cortex after a stimulation of at least a few seconds (Boynton, Engel, Glover, \& Heeger, 1996). Since the response time in our task is always longer than $2 \mathrm{sec}$, we have some reason to believe that the assumption of linearity is valid here as well; however, the available data are not sufficient to say so with certainty.

\section{Motor Activity During Mental Rotation}

The goal of our study was twofold. First, we wanted to determine if motor area activity occurs in relation to any aspect of this implementation of the mental-rotation task. We will assume that the multi-dimensional set of trapezoids used for parametric analysis (see Methods) is a reasonable approximation to the true activation shape. Even if this were not the case, false positives would occur only if there were a waveform present in the time courses that is indeed well approximated by this model, but not related to neuronal activation. Such a situation is conceivable for motion or physiological artifacts; however, the focal nature of the activation maps (see Figure 3) suggests that this is unlikely: We would expect both types of artifacts to be prevalent near large susceptibility gradients, found at the edge of the brain or near the ventricles. We conclude, then, that all seven areas under investigation are active in this task (with the possible exception of the right M1, which was found to be active in only five out of six subjects).

The second question probes the specific nature of the activation in relation to the execution of the task. Note that this task contains an overt motor component, since the subject presses a button at the end of the task period. All subjects used their right hand for this; hence, we expect the activation due to this component of the task to be mainly present in the left M1. This is, indeed, borne out by the observation that onset (but not width) and response time are generally correlated in that area, as shown in Table 2 (with the exception of one subject, which showed a correlation between the width and response time here). Note in this context that the statistical power of the experiment increases considerably when all six subjects are analyzed together; we concede, however, that it is implicitly assumed here that the hemodynamic response is similar in a given area of the brain between subjects. Furthermore, we cannot rule out in general that the left M1 is also involved in an aspect of the task that is not related to the button press. However, we cannot reject the null hypothesis that it is not involved, given the statistical power of this experiment. The same is true for the right M1; even though we see activation in some aspect of the task, we cannot determine, by this experiment, the details of its behavior.

The role of nonprimary-motor areas (here lateral and medial BA 6) and the parietal lobe is more obvious. Let us assume for the moment that our definition of the width (upslope plus plateau time) is a good predictor of the actual duration of neuronal activity. This, in turn, would be consistent with a model of the hemodynamic response, which assumes that the onset of neuronal activity triggers the increase in blood flow, that the end of the neuronal activity triggers the decrease in blood flow back to the baseline, and that the delay times are similar in both cases. This may be valid as long as the fMRI signal is within the linear domain, and the duration of stimulation is longer than the intrinsic rise time. A positive correlation between the width of the activation peak and the response time then means that the duration of the hemodynamic response in nonprimarymotor areas is a monotonically increasing and, maybe, linear function of the response time. This would mean in particular that the observed activation in those motor areas is likely related to the very execution of the mental-rotation task, and not only to an aspect of the task which is only peripherally related to mental rotation, such as, for example, the response selection or the actual pushing of the answer button at the end of the task. Another important issue is that of employing statistics across subjects. Ideally, we would like to use data from every subject individually. However, as shown in Table 1 , definite conclusions can only be reached for some of the subjects. Using all subjects simultaneously provides information about the beha- 
vior of a brain area in general, but information about individual subjects is lost, as, for example, the fact that in one subject the width, and not the onset of activation in the left M1, was correlated with the response time. This also assumes that the hemodynamic response times in a given area are similar across subjects. With these caveats, the combined statistics give valid information about the usual role of a given brain area in a complex task.

Some of the studies cited above did not find consistently, or at all, activation in motor areas during similar tasks; this discrepancy might be due to the differences in the specific paradigm, or to the measuring method employed. Most notable in this context is a study comparing the mental rotation of abstract objects and images of the hands (Kosslyn et al., 1998); a combined conclusion from that study and ours might be that the mental rotation of hand shapes activates the motor areas to a considerably greater extent than the mental rotation of Shepard and Metzler's objects. An important distinction among the tasks involving dynamic imagery is that of the internal and external perspectives (Isaac, Marks, \& Russell, 1986). If an internal perspective were assumed, the subjects might imagine themselves carrying out a movement, be it the movement of a body part or the manipulation of an object in space, or both. If an external perspective were assumed, the subject might imagine somebody else's manipulation of objects in space, or the motion of objects due to an inanimate or unspecified cause. It is possible that the involvement of the areas subserving overt movements in a given task of dynamic imagery might, in fact, be a function of the subject's perspective during the task, where these areas are active if an internal perspective is assumed. This would indeed imply that the subjects carry out this task by imagining that they themselves manipulate the objects in space. However, the external perspective model might still be consistent with our results, in light of the existence of mirror neurons (to primary-motor neurons) in area $\mathrm{F} 5$ of the macaque monkey, which are active during the observation of movements (Rizzolatti \& Fadiga, 1998). Additional experiments must be designed to separate the internal and external perspectives in the subjects. It must be conceded that the low-rotation speeds (and low-error rates) of our experiments suggest that the task used here, while still a task of mental rotation, is actually different from that of Shepard and Metzler's original experiment.

\section{METHODS}

\section{Subjects}

Eight normal subjects were studied according to the guidelines of the Institutional Review Board of the University of Minnesota. Informed consent was obtained from all participants. Six subjects (three men and three women, all right-handed) yielded fMRI data free of obvious motion artifacts; only these will be considered here.

\section{Task}

Before each study, the subjects practiced the mentalrotation task for 5 to 10 min outside of the magnet. The subjects then continued to practice the task inside the magnet during the setup period for the fMRI experiments. In these practice sessions, a pair of objects was shown until the subject pressed a button to indicate the decision; then the next pair of objects was shown.

For the fMRI studies, the images of the object pairs were projected onto a screen in the magnet and viewed by the subjects through a mirror. In the first period (baseline period: Duration $\approx 20 \mathrm{sec}$ ), two identical objects at the same view angle were shown. Then, one or both objects were replaced by different ones, signaling the beginning of the second period (task period: Duration $=$ response time). The new objects were, in general, shown rotated with respect to each other about their vertical axis. The subject decided whether the objects were identical or mirror images of each other, and indicated the decision by pressing one of two buttons on a key pad, using the right hand. As soon as a button was pressed, the objects reverted to the initial baseline state. During each trial, the task was performed once. Each experiment lasted approximately $60 \mathrm{sec}$. Response times were recorded for each trial. Each subject executed 16 trials in separate experiments with randomized objects and rotation angles, rotation angles ranged from $20^{\circ}$ to $80^{\circ}$; five different objects and their mirror images were used. No subject made more than two errors in the 16 experiments; the average error rate was 6 percent.

\section{Data Acquisition}

MRI experiments were carried out with a 4-T whole body imaging system with a homogeneous birdcage coil. In the first part of the session, anatomic images were acquired with a conventional TurboFLASH pulse sequence (Haase, 1990). From these images, four axial, 10-mm-thick slices were chosen for functional imaging. The four slices were contiguous, the topmost slice containing M1. Blood Oxygenation Level Development (BOLD)-based (Ogawa et al., 1992) functional images were then acquired with a single-shot or two-shot echoplanar imaging (EPI) sequence (echo time: 25 or 15 msec; repetition time (for four slices): 200 (one subject) or $480 \mathrm{msec}$; field of view: $(24 \times 24 \times 4) \mathrm{cm}^{3}$; matrix size: $64 \times 64 \times 4)$.

\section{Regions of Interest}

Regions of interest were drawn based on brain anatomy after a 3-D reconstruction of the multislice anatomical 
images, using both in-house software, and the software package STIMULATE (Strupp, 1996). For the purpose of this experiment, the regions were defined as follows: The SMA was defined as the medial part of BA 6, bordered caudally by the anterior lip of the precentral gyrus and laterally by the medial part of the superior frontal gyrus. The lateral-premotor area was defined as the lateral part of BA 6 bordered caudally by the midline of the precentral gyrus, rostrally by the anterior wall of the precentral sulcus, and medially by the lateral part of the superior-frontal gyrus. M1 was defined as the area bordered caudally by the anterior wall of the central sulcus and rostrally by the midline of the precentral gyrus. The SPL was defined as the area posterior to the postcentral sulcus, superior to the intraparietal sulcus, and lateral to the midline. Activations generally encompassed various other areas as well (as seen in Figure 3); however, only pixels wholly within these ROIs were considered for analysis.

\section{Data Analysis}

Time-resolved fMRI experiments cannot easily be analyzed by conventional (parametric) methods. The objective is to measure the temporal parameters of the activation peak and the response times independently from each other; hence, the activation maps should ideally be generated without bias toward a particular functional form of the activation. However, the a priori assessment of Type I errors is difficult in model-free methods, precisely because the functional form of the activation is not predefined and, hence, no hypothesis external to the data is tested. Thus, we used a modelfree method (Fuzzy Clustering Analysis, FCA) in combination with a conventional-multidimensional-correlation analysis to estimate Type I errors empirically.

For each subject, the 16 individual experiments were preprocessed in the following manner: First, images corresponding to the first $10 \mathrm{sec}$ of the experiments were removed to allow for the establishment of a steadystate condition of transverse magnetization. This resulted in 88 volumes per experiment (250 in one case). Then, drift was removed by a linear correction of each time course. No motion correction was carried out. Furthermore, time courses with a standard deviation of more than 3 percent were excluded from analysis so as to minimize the contributions from large vessels (Kim, Hendrich, Hu, Merkle, \& Ugurbil, 1994; Somorjai \& Jarmasz, 1999). No smoothing was performed.

\section{Fuzzy Clustering Analysis}

Our implementation of FCA (EvIdent ${ }^{(i x}$ v. 5.0, see www.ibd.nrc.ca/informatics/evident.html) is an extensively modified and improved version (Jarmasz \& Somorjai, 1998; Jarmasz \& Somorjai, in press; Somorjai, Jarmasz, Baumgartner, \& Richter, in press; Somorjai \& Jarmasz,
1999) of Bezdek's original algorithm (Bezdek, 1981; Scarth et al., 1995). This algorithm minimizes an objective function $J_{\mathrm{m}}(U, V)$ via a two-stage iterative process. At the first stage $K$ initial centroids $v_{k}$ are selected, using a deterministic algorithm (Tou \& Gonzales, 1974). $\mathbf{U}=\left\{\boldsymbol{u}_{k n}\right\}$ is the $K \times N$ matrix of fuzzy membership functions, $\boldsymbol{V}=\left\{v_{k}\right\}$ denotes the $K T$-dimensional centroid vectors, $m>1$ is the fuzzy index. The distance $d_{k n}$ is the Euclidean distance from the $n$th time course (TC) $x_{n}=\left\{x_{n 1}, x_{n 2}, x_{n 3}, \ldots, x_{n T}\right\}$ to the $k$ th cluster centroid $v_{k}=\left\{v_{k 1}, v_{k 2}, v_{k 3}, \ldots, v_{k T}\right\}$. One then calculates, for each voxel $x_{n}$, the cluster membership map $\left\{u_{k n}\right\}, 1 \leq n \leq N$ to each cluster $k, 1 \leq k \leq K$. After the cluster-membership map has been computed for all clusters, the second stage consists of updating the cluster centroids $v_{k}$. This twostage process is repeated until convergence is achieved.

Here, all 16 experiments were concatenated in time. For each ROI, EvIdent ${ }^{\circledR i}$ was asked to produce two and only two clusters. The expectation was that one of these would contain the activated voxel time courses (VTCs), whereas the other would consist of noise VTCs. The FC algorithm places VTC $x_{n}$ in cluster 1 if its membership value $u_{1 n}$ is $>.5$. We then calculated a correlation map between the cluster centroid and the VTCs making up the cluster. Only those VTCs were considered active that passed a correlation threshold, $r_{\text {thresh }}$, calculated from a preselected $p$ value (e.g., $p<.01$ after a Bonferroni correction for 16 repeated experiments) via the standard relation between Pearson's correlation coefficient $r$ and the $t$ value of Student's $t$ test.

\section{Parametric Analysis}

In order to assess Type I errors (and to produce the map shown in Figure 3), each VTC from each experiment was correlated with a set of trapezoids of varying onset, upslope, width, and downslope. Hence, our null hypothesis is that a given time course cannot be represented by such a trapezoid. The resulting correlation coefficients were averaged over the 16 trials after a Fisher $z$-transformation, and thresholded such that $p<.01$ after a Bonferroni correction for multiple comparisons (the set of trapezoids typically contained approximately 2000 members). Voxels found activated by this method were then compared to the voxels found activated by FCA. In each area, the overall false positive rate determined in this manner was below .05, with the exception of the right motor cortex, where the overall false positive rate was approximately .5. Hence, the trapezoidal model coincides largely with the result from FCA. The FCA analysis was faster by approximately four orders of magnitude than the parametric analysis.

\section{Correlation Between fMRI and Response Time}

Onset and width were approximated by a multidimensional fit with a set of trapezoids of varying onset, rise 


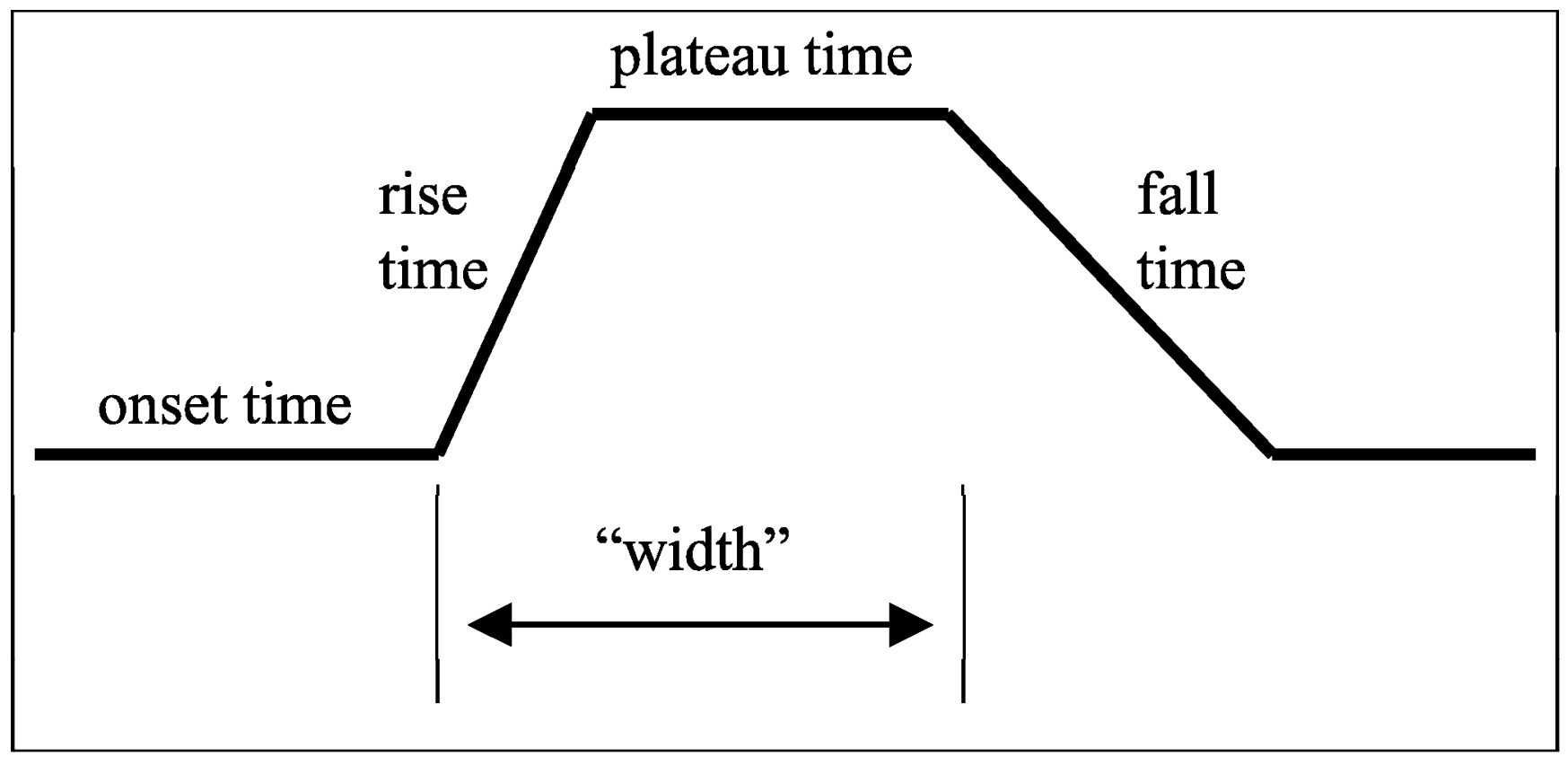

Figure 7. Trapezoidal function used to fit the activation peaks. The four variable parameters are shown. The parameter "width" is defined as the sum of the rise time and the plateau time.

time, plateau time, and fall time; we defined the width as the sum of the rise time and the plateau time (Figure 7). An example is given in Figure 8; shown are the time courses from the 81 activated voxels in the right premotor area for two experiments, together with the trapezoidal fits. Note that the onset is independent of response time, whereas the width is a monotonic function of the response time. Onset time and width were correlated with response times. Then the confidence levels for the null hypothesis that the onset or width is not correlated with response times were calculated from Pearson's correlation coefficient.
Figure 8. Trapezoidal fit to two time courses (from two different trials, identical pixels in the right premotor area). The two response times are marked by vertical lines.

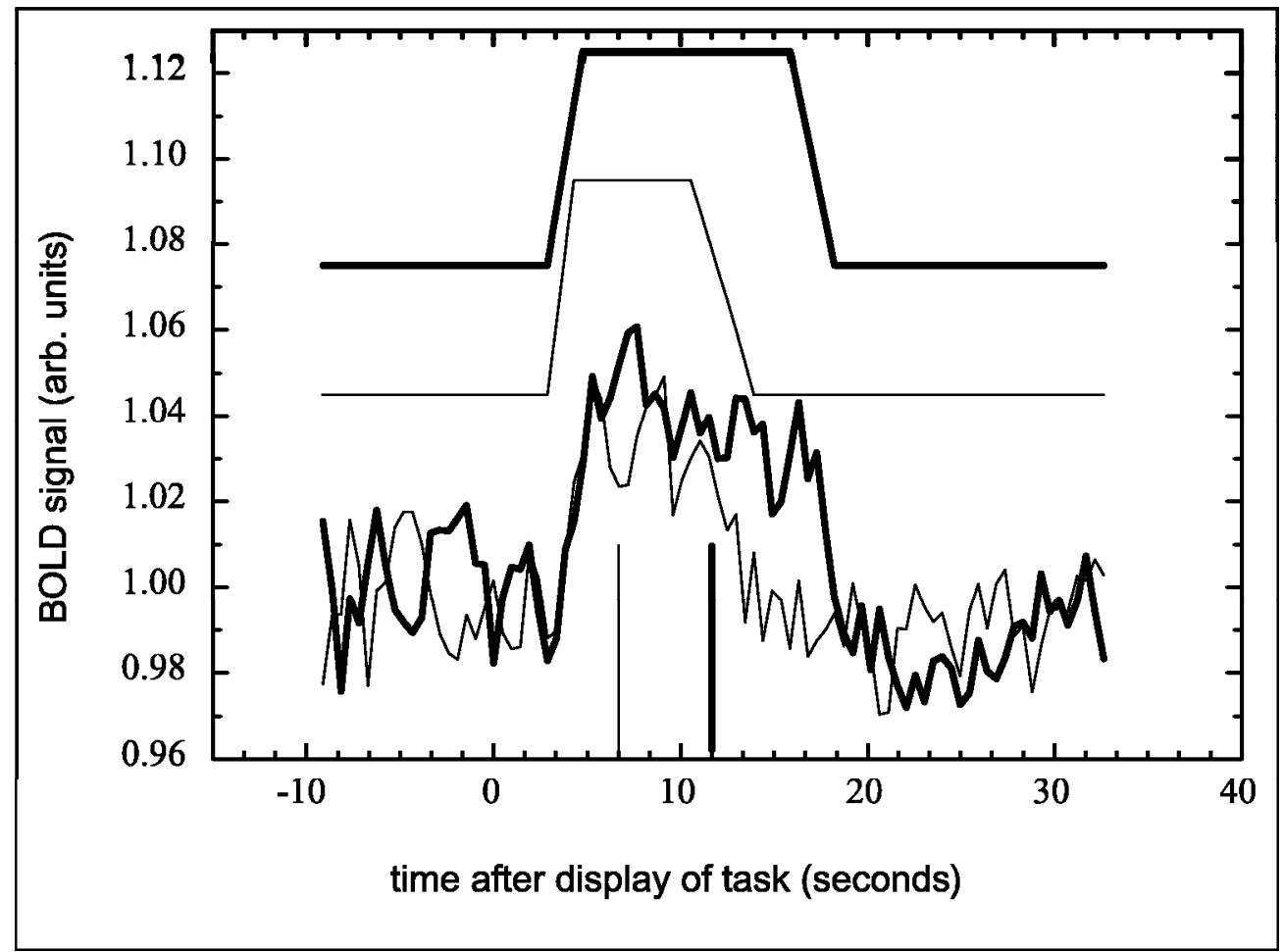




\section{Acknowledgments}

The authors thank Dr. Peter Andersen and Dr. Gregor Adriany for hardware support. This project was supported by $\mathrm{NIH}$ grants MH57180, RR08079 and NS32919.

Reprint requests should be sent to: Wolfgang Richter, Conseil national de recherches Canada, Institut du biodiagnostic, 435, avenue Ellice, Winnipeg MB, Canada R3B 1Y6.

\section{REFERENCES}

Alexander, G. E., \& Crutcher, M. D. (1990). Preparation for movement: Neural representations of intended direction in three motor areas of the monkey. Journal of Neurophysiology, 64, 133-150.

Alivisatos, B., \& Petrides, M. (1996). Functional activation of the human brain during mental rotation. Neuropsychologia, 35, 111-118.

Bezdek, J. (1981). Pattern recognition with fuzzy objective function algorithms. New York: Plenum.

Boynton, G. M., Engel, S. A., Glover, G. H., \& Heeger, D. J. (1996). Linear systems analysis of functional magnetic resonance imaging in human V1. Journal of Neuroscience, 16, 4207-4221.

Buckner, R. L., Bandettini, P. A., O'Craven, K. M., Savoy, R. L., Petersen, S. E., Raichle, M. E., \& Rosen, B. R. (1996). Detection of cortical activation during averaged single trials of a cognitive task using functional magnetic resonance imaging. Proceedings of the National Academy of Science, U.S.A., 93, $14878-14883$.

Clark, V. P., Maisog, J. M., \& Haxby, J. V. (1998). fMRI study of face perception and memory using random stimulus sequences. Journal of Neurophysiology, 79, 20892-21366.

Cohen, M. S., Kosslyn, S. M., Breiter, H. C., DiGirolamo, G. J., Thompson, W. L., Anderson, A. K., Bookheimer, S. Y., Rosen, B. R., \& Belliveau, J. W. (1996). Changes in cortical activity during mental rotation. A mapping study using functional MRI. Brain, 119, 89-100.

Georgopoulos, A. P., Masato, T., \& Lukashin, A. (1993). Cognitive neurophysiology of the motor cortex. Science, 260, 47-52.

Haase, A. (1990). Snapshot FLASH MRI. Applications to T1, T2 and chemical-shift imaging. Magnetic Resonance in Medicine, 13, 77-89.

Isaac, A., Marks, D., \& Russell, D. (1986). An instrument for assessing imagery of movement: The vividness of movement imagery questionnaire (VMIQ). Journal of Mental Imagery, 10, 23-30.

James, W. (1950). The principles of Psychology. New York: Dover.

Jarmasz, M., \& Somorjai, R. L. (1998). Time to join! Cluster merging in unsupervised fuzzy clustering of functional MRI data. In Abstracts: Sixth annual meeting ISMRM (p. 2068). Sydney, Australia.

Jarmasz, M., \& Somorjai, R. L. (in press). Crossing the median: Separating potential fMRI activations from noise. In $A b$ stracts: Seventh annual meeting ISMRM. Philadelphia, USA.

Kim, S.-G., Hendrich, K., Hu, X., Merkle, H., \& Ugurbil, K. (1994). Potential pitfalls of functional MRI using conventional gradient-recalled echo techniques. NMR in Biomedicine, 7, 69-74.

Kim, S.-G., Richter, W., \& Ugurbil, K. (1997). Limitations of temporal resolution in functional magnetic resonance imaging. Magnetic Resonance in Medicine, 37, 631.

Kosslyn, S. M., Digirolamo, G. J., Thompson, W. L., \& Alpert, N. M. (1998). Mental rotation of objects versus hands: Neural mechanisms revealed by positron emission tomography. Psychophysiology, 35, 151-161.
Lee, A. C., Harris, J. P., \& Calvert, J. E. (1997). Impairments of mental rotation in Parkinson's disease. Neuropsychologia, 36, 109-114.

Menon, R. S., \& Kim, S.-G. (1999). Spatial and temporal limits in cognitive neuroimaging by fMRI. Trends in Cognitive Sciences, 3, 207-216.

Menon, R. S., Lucknowsky, D. C., \& Gati, J. S. (1998). Mental chronometry using latency-resolved functional MRI. Proceedings of the National Academy of Science, U.S.A., 95, 10902-10907.

Ogawa, S., Tank, D. W., Menon, R., Ellermann, J. M., Kim., S.-G., Merkle, H., \& Ugurbil, K. (1992). Intrinsic signal changes accompanying sensory stimulation: Functional brain mapping with magnetic resonance imaging. Proceedings of the National Academy of Science, U.S.A., 89, 5951-5955.

Richter, W. (in press). High temporal resolution fMRI at high field. Topics in Magnetic Resonance Imaging.

Richter, W., Andersen, P. M., Georgopoulos, A. P., \& Kim, S.-G. (1997a). Sequential activity in human motor areas during a delayed cued finger movement task studied by time-resolved fMRI. NeuroReport, 8, 1257-1261.

Richter, W., Ugurbil, K., Georgopoulos, A., \& Kim, S.-G. (1997b). Time-resolved fMRI of mental rotation. NeuroReport, 8, 3697-3702.

Rizzolatti, G., \& Fadiga, L. (1998). Grasping objects and grasping action meanings: The dual role of monkey rostroventral premotor cortex (area F5). Novartis Foundation Symposium, 218, 81-95.

Scarth, G., Somorjai, R., Alexander, M., Wowk, B., Wennerberg, A. W. A., \& McIntyre, M. C. (1995). Cluster analysis of functional brain images. Human Brain Mapping, S1, 158.

Shepard, R. N., \& Metzler, J. (1971). Mental rotation of threedimensional objects. Science, 171, 701-703.

Somorjai, R. L., \& Jarmasz, M. (1999). Exploratory data analysis of fMR images: Philosophy, strategies, tools, implementation. In Abstracts: Seventh annual meeting ISMRM. Philadelphia, USA, p. 1714.

Somorjai, R. L., Jarmasz, M., Baumgartner, R., \& Richter, W. (1999). Exploratory analysis of fMR images: Voxel preselection via "self-similarity." In Abstracts: Seventh annual meeting ISMRM. Philadelphia, USA, p. 1718.

Strupp, J. P. (1996). Stimulate: A GUI-based fMRI analysis software package. Neuroimage, 3, S603.

Tagaris, G. A., Kim, S.-G., Strup, J. P., Andersen, P., Ugurbil, K., \& Georgopoulos, A.P. (1996). Quantitative relations between parietal activation and performance in mental rotation. NeuroReport, 7, 773-776.

Tagaris, G. A., Kim, S. G., Strupp, J. P., Andersen, P., Ugurbil, K., \& Georgopoulos, A. P. (1997). Mental rotation studied by functional magnetic resonance imaging at high field ( 4 Tesla): Performance and cortical activation. Journal of Cognitive Neuroscience, 9, 419-432.

Tagaris, G. A., Richter, W., Kim, S. G., Pellizzer, G., Andersen, P., Ugurbil, K., \& Georgopoulos, A. P. (1998). Functional magnetic resonance imaging of mental rotation and memory scanning: A multidimensional scaling analysis of brain activation patterns. Brain Research Reviews, 26, 106-112.

Tou, J. C., \& Gonzales, R. C. (1974). Pattern recognition principles. Reading, MA: Addison-Wesley.

Weimer, W. B. (1977). A conceptual framework for cognitive psychology: Motor theories of the mind. In R. Shaw \& J. Bransford (Eds.), Perceiving, acting, and knowing. Hillsdale, NJ.

Williams, J. D., Rippon, G., Stone, B. M., \& Annett, J. (1995). Psychophysiological correlates of dynamic imagery. British Journal of Psychology, 86, 283-300.

Zarahn, E., Aguirre, G. K., \& D'Esposito, M. (1997). A trial-based experimental design for fMRI. Neuroimage, 6, 122-138. 